\title{
RELAÇÃO ENTRE APTIDÃO FISICA, QUALIDADE DE VIDA E SINTOMATOLOGIA DEPRESSIVA DE IDOSOS FISICAMENTE ATIVOS
}

\author{
Vanessa Dias Possamai ${ }^{1}$ \\ Amanda Suely Rodrigues de Vargas ${ }^{2}$ \\ Priscilla Cardoso da Silva ${ }^{3}$ \\ Valéria Feijó Martins ${ }^{4}$ \\ Andréa Krüger Gonçalves ${ }^{5}$
}

resumo

O objetivo deste estudo foi verificar a relação entre as variáveis de aptidão física, qualidade de vida e sintomatologia depressiva

\footnotetext{
1 Graduada em Educação Física. Doutoranda em Ciências do Movimento Humano na Universidade Federal do Rio Grande do Sul (UFRGS). E-mail: vanessa.possamai@ufrgs.br.

2 Graduada em Licenciatura em Educação Física. Mestre em Saúde Coletiva, Universidade Federal do Rio Grande do Sul na Universidade Federal do Rio Grande do Sul (UFRGS). E-mail: amanda.rvargas2@gmail.com.

3 Graduada em Bacharelado em Fisioterapia e Educação Física. Graduada em Licenciatura em Educação Física. Mestranda em Ciências do Movimento Humano, Universidade Federal do Rio Grande do Sul (UFRGS). E-mail: priscilla.cardosos@gmail.com.

4 Graduada em Educação Física. Doutoranda em Ciências do Movimento Humano na Universidade Federal do Rio Grande do Sul (UFRGS). E-mail: valeriafeijo4@gmail.com.

5 Graduada em Educação Física. Prof. ${ }^{a}$ Dr. ${ }^{a}$ da Escola de Educação Física, Fisioterapia e Dança da Universidade Federal do Rio Grande do Sul (UFRGS). E-mail: andreakgoncalves@gmail.com.
} 
em idosos fisicamente ativos. Participaram do estudo 135 idosos participantes de um programa de extensão universitária que oferece atividades físicas e socioeducativas, duas a quatro vezes por semana. Foi utilizado o questionário GDS-15 para avaliar a sintomatologia depressiva, SF-36 para a qualidade de vida e a bateria Senior Fitness Test para avaliar a aptidão física. Utilizou-se estatística descritiva para a análise dos resultados, com média e desvio padrão, além de Teste de Pearson para relacionar as variáveis ( $p \leq 0.05)$. Houve correlação significante da qualidade de vida com todas as variáveis de aptidão física e os sintomas depressivos. Também ocorreu correlação entre todos os domínios do SF-36 com o GDS. Programas de atividade física que promovam a melhorar ou manutenção das aptidões físicas podem contribuir positivamente na sintomatologia depressiva e na qualidade de vida de idosos, tanto pelo engajamento social quanto pelo estímulo físico.

palavras-chave

Idoso. Aptidão Física. Qualidade de Vida. Depressão.

Na medida em que as condições gerais de vida e o avanço da ciência têm contribuído para controlar e tratar muitas doenças responsáveis pela mortalidade, a expectativa de vida da população, tanto dos países desenvolvidos como da maioria dos países em desenvolvimento, tem aumentado nesses últimos anos (MACIEL et al., 2013). Segundo o Instituto Brasileiro de Geografia e Estatística, o crescimento da população idosa, em números absolutos e relativos, é um fenômeno mundial e está ocorrendo a um nível sem precedentes, estima-se que em 2050 um quinto da população será de pessoas idosas (IBGE, 2010).

Ao longo do processo de envelhecimento, ocorrem modificações morfológicas, fisiológicas, bioquímicas e psicológicas, as quais determinam a perda progressiva da capacidade de adaptação do indivíduo ao meio ambiente (FERREIRA et al., 2010). De acordo com Farias (2010), as reservas funcionais dos indivíduos tendem a diminuir. As comorbidades podem levar o idoso a condições incapacitantes, isolamento social e também depressão.

Diante do aumento da longevidade e de todas mudanças biopsicossociais que acompanham o envelhecimento, a preocupação com a qualidade de vida e o surgimento de doenças crônicas, especialmente a depressão, tem demandado 
maior atenção (GONÇALVES et al., 2014). Entretanto, parte dos efeitos da senescência pode ser atenuada de acordo com o estilo de vida adotado pelas pessoas ao longo dos anos. Desse modo, um desafio é a conquista da velhice com qualidade de vida e condições de saúde (PASCHOAL, 2011).

Diante de tal realidade, torna-se fundamental a manutenção de padrões positivos de saúde e qualidade de vida, garantindo que a conquista da longevidade possa ser acompanhada a níveis satisfatórios de autonomia e independência (FERRETTI et al., 2015). A qualidade de vida é definida pela Organização Mundial de Saúde (WHO), como a sendo "a percepção que o indivíduo tem de sua posição na vida dentro do contexto de sua cultura e do sistema de valores de onde vive, e em relação a seus próprios objetivos, expectativas, bem como padrões e preocupações" (WHO, 1996, p. 1405).

A adoção de hábitos saudáveis, como, por exemplo, a prática de atividade física, promove a saúde ao longo da vida, influenciando o envelhecimento de maneira positiva nas variáveis físicas, psicológicas, sociais e ambientais (MERQUIADES et al., 2009). A prática regular de exercício físico para as pessoas idosas melhora a capacidade respiratória, reserva cardíaca, força muscular, memória recente, cognição e habilidades sociais, manutenção ou melhora de índices de doenças crônicas, além dos níveis de aptidão física e capacidade funcional (SILVEIRA et al., 2011). Salienta-se, ainda, a importância da prática de exercício como prevenção, ou seja, atuando como um fator protetor para o aparecimento de doenças crônicas (PEREIRA; NOGUEIRA; SILVA, 2015).

O objetivo deste estudo é verificar a relação entre qualidade de vida, aptidão física e a sintomatologia depressiva em idosos fisicamente ativos.

\section{Métodos}

O estudo realizado foi do tipo transversal. A amostra foi composta por 135 idosos participantes de um programa de extensão universitária que oportuniza exercícios físicos organizados em diferentes modalidades como ginástica funcional, hidroginástica, jogging aquático, musculação, dança, equilíbrio e PAIF (promovendo autonomia e independência funcional) com idade média de 72,8 $( \pm 7,5)$ anos. O tempo médio de participação no programa de extensão é de 8,3 $( \pm 4,9)$ anos. As atividades são realizadas duas vezes por semana, sendo que os idosos podem realizar até duas destas (totalizando quatro dias na semana). Os idosos foram avaliados após nove meses de intervenção.

Os critérios de inclusão foram: não possuir contra indicações para o exercício físico, participar de no mínimo $70 \%$ do programa físico; os critérios de 
exclusão foram: limitações físicas que interferissem na marcha, qualquer tipo de restrição musculoesquelética ou problemas neurológicos que comprometesse a realização dos exercícios ou a avaliação.

Para mensurar os sintomas de depressão, foi utilizado o protocolo GDS-15 (Geriatric Depression Scale) (SHEIKH; YESAVAGE, 1986), a partir do somatório das quinze questões. De acordo com O. Almeida e S. Almeida (1999), a pontuação entre 5 e 6 indica caso e não caso, ou seja, inferior significa que não há presença de sintomatologia depressiva e igual ou superior que há presença de sintomas. Para a avaliação da qualidade de vida, foi aplicado o questionário SF-36 - "The Medical Outcomes Study 36 - Item Short Form Health Survey" (CICONELLI, 1997). Este instrumento é um questionário multidimensional, formado por 36 itens que englobam oito domínios: capacidade funcional (CF), aspectos físicos (AF), dor (DOR), estado geral da saúde (EGS), vitalidade (VIT), aspectos sociais (AS), aspectos emocionais (AE) e saúde mental (SM). A pontuação máxima para cada domínio é 100, sendo que a soma total é no máximo 150, sendo zero "pior" e 150 "melhor estado de saúde". No nosso estudo, utilizou-se o somatório do SF-36.

Para avaliação da aptidão física foi utilizado o Senior Fitness Test proposto por Rikli e Jones (1999). A bateria é composta por seis testes que avaliam força, flexibilidade, resistência aeróbica, equilíbrio e agilidade. Para avaliar a flexibilidade de membros superiores (FLEXMS), os indivíduos são submetidos ao teste "alcançar atrás das costas", para flexibilidade de membros inferiores (FLEXMI) o teste "sentado e alcançar", ambos mensurados em centímetros. Para avaliar equilíbrio e agilidade (EA) os indivíduos realizaram o teste "levantar e caminhar $2,44 \mathrm{~m}$ ", o resultado é dado pelo tempo que o indivíduo demora a fazer o percurso. Para medir a força de membros superiores (FMS) foi realizado o teste "flexão de antebraço" e o teste "sentar e levantar" para medir força de membros inferiores (FMI) para verificar quantas repetições o indivíduo consegue fazer em 30 segundos. Para avaliar a resistência aeróbia (RA) os indivíduos realizaram o teste de "marcha estacionária de dois minutos de step no próprio lugar".

O tratamento estatístico incluiu análise descritiva com cálculo de frequência e percentual para as variáveis de perfil sociodemográfico, seguido de teste de correlação de Pearson (nível de significância de 95\%, p < 0,05) para verificar a associação entre as variáveis de aptidão física, sintomas depressivos e qualidade de vida. As análises de dados foram realizadas por meio do software estatístico SPSS, versão 22.0. O projeto foi aprovado pelo Comitê de Ética em Pesquisa da Universidade (parecer no 870.096), respeitando as diretrizes da Resolução 466/2012 do Conselho Nacional de Saúde, a qual trata de pesquisa com seres humanos. Foram obtidas as assinaturas do Termo de Consentimento Livre e Esclarecido de todos os participantes do estudo. 
Tabela 1 - Distribuição de frequência e percentual das características sociodemográficas dos participantes do programa de atividade física para idosos.

\begin{tabular}{|c|c|c|}
\hline Variáveis & $\mathrm{N}$ & $\%$ \\
\hline \multicolumn{3}{|l|}{ Sexo } \\
\hline Feminino & 118 & 87,4 \\
\hline Masculino & 17 & 12,6 \\
\hline \multicolumn{3}{|l|}{ Estado Civil } \\
\hline Casado & 59 & 43,7 \\
\hline Viúvo & 41 & 30,4 \\
\hline Divorciado & 25 & 18,5 \\
\hline Solteiro & 10 & 7,4 \\
\hline \multicolumn{3}{|l|}{ Moradia } \\
\hline Cônjuge & 58 & 43,0 \\
\hline Sozinho & 26 & 19,3 \\
\hline Cônjuge e outros & 41 & 30,4 \\
\hline Outros familiares & 10 & 7,4 \\
\hline \multicolumn{3}{|l|}{ Ocupação } \\
\hline Aposentado & 102 & 75,5 \\
\hline Ainda trabalha & 25 & 18,5 \\
\hline Nunca trabalhou & 8 & 5,9 \\
\hline \multicolumn{3}{|l|}{ Renda Mensal } \\
\hline 1 a 3 SM & 29 & 21,5 \\
\hline 4 a 6 SM & 56 & 41,5 \\
\hline 7 ou mais SM & 50 & 37 \\
\hline \multicolumn{3}{|l|}{ Escolaridade } \\
\hline 1 a 3 anos & 23 & 17 \\
\hline 4 a 7 anos & 64 & 47,4 \\
\hline 8 anos ou mais & 48 & 35,6 \\
\hline
\end{tabular}

Legenda: SM = salário mínimo.

Fonte: Elaborada pelas autoras, 2018 
De acordo com a Tabela 1, verifica-se que há maior participação de mulheres $(87,4 \%)$, a maioria casadas $(43,7 \%)$, e residem com o cônjuge (43\%). Em relação à ocupação $75,5 \%$ são aposentadas, mas 18,5\% ainda trabalham. A renda mensal predominante foi de 4 a 6 salários mínimos (47,4\%) e a escolaridade foi de 4 a 7 anos de estudo (47,4\%).

Tabela 2 - Média e desvio-padrão das variáveis de aptidão física, qualidade de vida e sintomatologia depressiva.

\begin{tabular}{lccc}
\hline Variável & N & Média & Desvio-padrão \\
\hline Aptidão Física & & & \\
FMl (repetições) & 134 & 19,28 & 6,14 \\
FMS (repetições) & 134 & 22,91 & 5,18 \\
FlexMl (cm) & 135 & 1,80 & 11,47 \\
EA (segundos) & 135 & 4,89 & 1,89 \\
FLEXMS (cm) & 135 & $-5,91$ & 10,12 \\
RA (repetições) & 134 & 90,35 & 22,92 \\
Qualidade de vida & & & \\
CF & 116 & 73,49 & 21,75 \\
AF & 114 & 67,98 & 40,25 \\
AM & 115 & 75,36 & 35,62 \\
DOR & 115 & 65,33 & 23,53 \\
SM & 116 & 79,86 & 17,67 \\
VIT & 116 & 71,77 & 18,13 \\
AS & 116 & 82,86 & 22,14 \\
EGS & 116 & 17,78 & 3,28 \\
Soma SF Total & 116 & 122,55 & 17,83 \\
GDS & 115 & 2,43 & 2,55 \\
GDS & & & \\
\hline
\end{tabular}

Legenda: FMl = Força de Membro Inferior; FMS = Força de Membro Superior; FLEXMl = Flexibilidade de Membro Inferior; EA: Equilíbrio e Agilidade; FLEXMS = Flexibilidade de Membro Superior; $\mathrm{RA}=$ Resistência Aeróbica; $\mathrm{CF}=$ Capacidade Funcional; $\mathrm{AF}=$ Aspectos Físicos; $\mathrm{AE}=$ Aspectos Emocionais; DOR = Dor; SM = Saúde Mental; VIT = Vitalidade; $\mathrm{AS}=$ Aspectos Sociais; EGS = Estado Geral de Saúde; SF Total = Soma Total Qualidade de Vida; GDS = Sintomatologia Depressiva.

Fonte: Elaborada pelas autoras, 2018. 
A Tabela 2 mostra as médias e desvio-padrão obtidos pelos idosos após nove meses de intervenção no programa de exercício físico multicomponente. Na avaliação de FMI e FMS os idosos apresentaram média de $19,28( \pm 6,14)$ repetições e 22,91 $( \pm 5,18)$ repetições, respectivamente. Na FLEXMI a média foi de 1,80 $( \pm 11,47)$ centímetros e na FLEXMS $-5,91( \pm 10,12)$ centímetros. No EA, a média foi de $4,89( \pm 1,89)$ segundos e na RA a média foi de $90,35( \pm 22,92)$ repetições.

No estudo de Vila et al. (2013) foram avaliados idosos praticantes de atividade física através do Senior Fitness Test. As médias encontradas foram inferiores à deste estudo nos testes de FMI e FMS (12 e 15 repetições), assim como o tempo de execução no teste de EA (6,08 segundos), e na FLEXMS $(-12,42 \mathrm{~cm})$. Mas no teste de FLEXMI apresentou média superior $(2,67 \mathrm{~cm})$.

Ainda, em nosso estudo, a avaliação da qualidade de vida apresentou média de $122,55( \pm 17,83)$ pontos. Ressalta-se que a pontuação máxima do questionário é de 150 pontos, demonstrando uma pontuação satisfatória. O estudo de Queiroz et al. (2016), com idosos fisicamente ativos encontrou pontuação de 73,3 no SF-36, pontuação inferior ao nosso estudo.

Os domínios que apresentaram maior pontuação média no SF-36 foram aspectos sociais $(83 \pm 22)$ e saúde mental $(80 \pm 17)$, seguido por aspectos emocionais $(75 \pm 35)$ e capacidade funcional $(73 \pm 22)$. Corroborando com este estudo, Queiroz et al. (2016) também encontraram escores mais elevados nos domínios de aspectos sociais e saúde mental de idosos que praticavam atividade física. Estes resultados podem estar relacionados com as atividades oferecidas no programa, já que são realizadas em grupo, o que facilita o envolvimento social e contato com pessoas da mesma faixa etária, fatores significativos para aumentar o suporte social do idoso.

No estudo de Toscano e Oliveira (2009) com 238 idosas de um grupo de convivência divididas pelo nível de atividade física, verificaram através do SF-36 que os domínios que apresentaram melhores escores foram: vitalidade, aspectos físicos, aspectos emocionais e aspectos sociais. Ao comparar os diversos domínios da qualidade de vida entre os grupos de idosas mais ativas com as menos ativas, verificou-se que os resultados dos indivíduos mais ativos foram significativamente superiores em todos os domínios. Esses achados se assemelham com os resultados em nosso estudo, onde os idosos participantes de atividades físicas em grupo, apresentaram melhores resultados em variáveis relacionadas aos aspectos sociais, físicos e emocionais. 
$\mathrm{Na}$ avaliação da sintomatologia depressiva a média apresentada foi de $2,43( \pm 2,55)$ pontos, indicando ausência de sintomas depressivos nesta amostra. Em um estudo realizado por Mazo et al. (2012) com 174 idosos praticantes de exercício físico apenas 9,2\% dos idosos apresentaram diagnóstico de depressão. Para Stella et al. (2002) a atividade física contribui de diferentes maneiras para melhorar a condição clínica geral e a condição mental do idoso deprimido. Do ponto de vista biológico, a atividade física tem sido associada a vários fatores favoráveis a uma melhor qualidade de vida no idoso, além de elevar a autoestima, melhorar relações psicossociais e estimula as funções cognitivas. A Tabela 3, a seguir, apresenta a correlação entre as variáveis de aptidão física com os domínios do SF-36 e sintomas depressivos. 


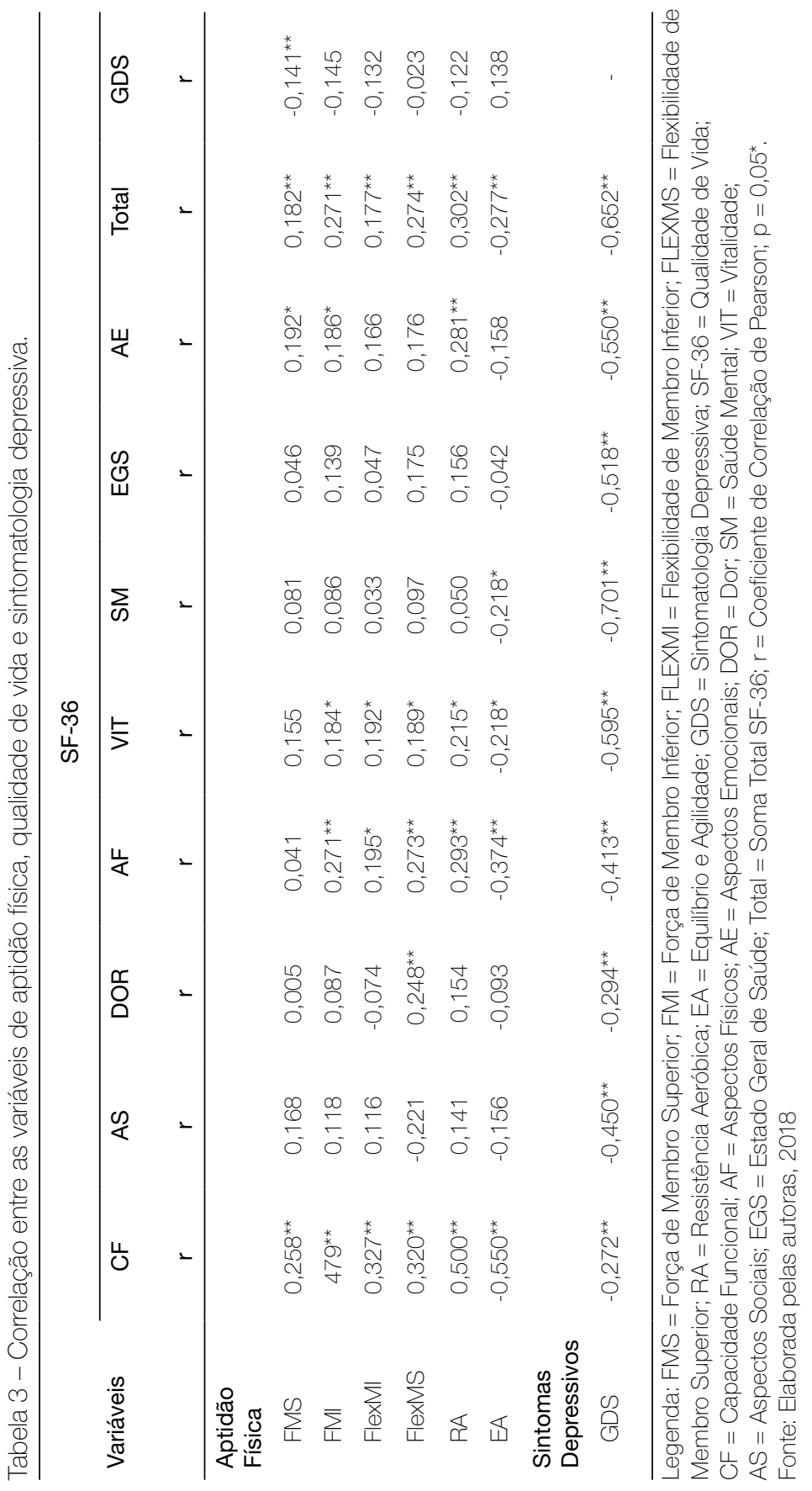

0
0
$\circlearrowleft$
$\square$
$\square$
$\square$

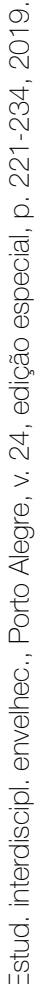


De acordo com a Tabela 3, a análise estatística indicou que houve correlação significante entre todas as variáveis de aptidão física (FLEXMI, FLEXMS, FMI, FMS, RA e EA) com o somatório total do questionário de qualidade de vida (SF-36). Na análise realizada entre as variáveis de aptidão física e domínios da qualidade de vida destaca-se que houve correlação entre todas as variáveis da aptidão física com o domínio de capacidade funcional. A força de membros inferiores também apresentou correlação com o domínio de aspectos físicos, vitalidade e aspectos emocionais. Flexibilidade de membros inferiores apresentou correlação com o domínio de aspectos físicos e vitalidade. Flexibilidade de membros superiores teve correlação com dor, aspectos físicos, e vitalidade. Na resistência aeróbica houve correlação entre aspectos físicos, vitalidade e aspectos emocionais. No equilíbrio e agilidade houve correlação com aspectos físicos, vitalidade e saúde mental.

No teste de correlação realizado por domínios do SF-36 com o GDS a análise estatística indicou que houve correlação do GDS com todos os domínios da qualidade de vida (CF, AF, AE, DOR, SM, VIT, AS, EGS). Além disso, também houve correlação significante no somatório total do questionário com a sintomatologia depressiva.

Não ocorreu correlação significante entre as variáveis de aptidão física e sintomatologia depressiva, mesmo a que a média do GDS tenha indicado ausência de sintomas depressivos. De acordo com Silva (2013), a atividade física pode ser fundamental para a melhora da sintomatologia depressiva, ainda, quando praticada regularmente auxiliam na prevenção de outras doenças de origem degenerativa, além de contribuir para a manutenção do desempenho cognitivo, possibilitando à socialização e integração.

A prática de atividade física regular por idosos pode ser considerado um fator importante para a diminuição de sintomatologia depressiva melhorando sua qualidade de vida. No estudo de Mather et al. (2002) foi encontrada redução significativa de 55\% dos níveis de depressão, quando submeteram 86 idosos a aulas de ginástica coletiva durante 12 semanas. Destacando que os idosos deste estudo praticam atividade física regular duas vezes por semana no mínimo. De outra forma, a relação entre a atividade física e depressão pode ser estudada em duas vertentes distintas. Em primeiro lugar, é importante considerar que a depressão pode reduzir a prática de atividades físicas, uma vez que o idoso tende a ficar mais isolado e a não sair de casa. Por outro lado, a atividade física pode ser um coadjuvante na prevenção e no tratamento da depressão no idoso (MORAES et al., 2007).

Guimarães et al. (2012) investigaram a associação entre qualidade de vida e atividade física. Os resultados mostram uma relação direta e positiva 
de interdependência entre essas duas variáveis, ou seja, melhor qualidade de vida leva a maior prática de atividade física de idosos e vice-versa. A prática de exercício físico prepara o idoso para uma vida autônoma e independente. Lago e Reis (2017) avaliaram a relação entre qualidade de vida e desempenho motor em idosos ativos. Foi encontrada diferença significativa nos domínios da qualidade de vida com o equilíbrio. Para as autoras, a qualidade de vida pode interferir de forma direta no desempenho motor dos idosos, uma vez que a qualidade de vida determina o bem-estar e a capacidade de manter-se autônomo para realizar as atividades diárias.

Gonçalves et al. (2014) verificaram a diferença entre sintomas depressivos e qualidade de vida a partir dos questionários GDS e SF-36, em idosos de diferentes faixas etárias praticantes de atividade física. Não foram encontradas diferenças significantes entre os grupos, porém em todas as faixa etárias apresentaram qualidade de vida satisfatória e ausência de sintomas depressivos.

Haider et al. (2016), apontam que o exercício físico é capaz de influenciar e reduzir o risco de desenvolvimento de doenças crônicas, incluindo depressão, doenças cardiovasculares, hipertensão, osteoporose, obesidade, comprometimento cognitivo, bem como pode aumentar a capacidade aeróbica, a força muscular e outras variáveis da aptidão física. Outros estudos (PATROCINIO; TORRES; GUARIENTO, 2013; NASCIMENTO, C. et al., 2013; NASCIMENTO, A. et al., 2013) relatam que a prática de exercícios físicos está ligada com sensações de bem-estar psicológico, melhora da autoestima, autoimagem, saúde mental e qualidade de vida. Idosos praticantes de exercícios físicos regulares apresentam menos casos de depressão, sobretudo quando realizados em conjunto com pessoas de idade ou patologias semelhantes, em que ocorre grande socialização, surgindo novos interesses.

Em um estudo de revisão sistemática realizado por Oliveira et al. (2010), verificou-se que os índices de qualidade de vida de idosos aumentam quando esses indivíduos participam de programa com exercícios na água, treino aeróbio e de força. Ainda, constataram que a prática de exercícios com frequência de duas vezes por semana parece ser mais benéfica para a qualidade de vida do que a prática realizada apenas uma vez por semana. Esses dados que se assemelham aos deste estudo, pois os idosos da amostra realizavam exercício físico de duas a quatro vezes por semana, com atividades que variam entre hidroginástica, ginástica funcional, jogging aquático, equilíbrio, dança e musculação.

Sendo assim, a participação em atividades em grupos, como os programas públicos de exercícios físicos, assume um papel importante na qualidade de vida dos idosos, pois também podem contribuir para que a pessoa idosa se sinta agente participante da vida em comunidade. 
Conclui-se que em relação a avaliação de qualidade de vida, houve correlação significante com a presença dos sintomas depressivos, e com todas as variáveis de aptidão física (FMI, FMS, FLEXMI, FLEXMS, EA, RA). Além disso, no teste de correlação realizado por domínios do SF-36, a análise estatística indicou que houve correlação entre todos os domínios do questionário com o GDS.

A partir dos resultados e estudos indicados, verificamos que existe uma associação positiva entre as variáveis de aptidão física como a melhora da sintomatologia depressiva e acarretando uma melhora na qualidade de vida consequentemente. Sugere-se que programas de atividade física podem contribuir positivamente na qualidade de vida na população de idosos, tanto pelo engajamento social quanto pelo estímulo físico, resultando em maior autonomia. Além disso, as alterações inerentes ao processo de envelhecimento são minimizadas por meio da prática de exercícios físicos.

Sugerem-se novos estudos acerca desta temática, principalmente na relação dos componentes da aptidão física com sintomas depressivos ao longo do tempo.

RELATIONSHIP BETWEEN PHYSICAL FITNESS, LIFE QUALITY AND DEPRESSIVE SYMPTOMS OF OLD PRACTICERS OF PHYSICAL ACTIVITY

\section{abstract}

The objective of this study was to verify the relationship between the variables of physical fitness, quality of life and depressive symptomatology in physically active elderly. The study was attended by 135 elderly participants of a university extension program that offers physical and socio-educational activities two to four times a week. The GDS-15 questionnaire was used to evaluate the depressive symptomatology, SF-36 for quality of life and the Senior Fitness Test battery to assess physical fitness. Descriptive statistics were used to analyze the results, with mean and standard deviation, as well as Pearson's test to relate the variables $(p \leq 0.05)$. There was a significant correlation of quality of life with all physical fitness variables and depressive symptoms. There was also a correlation between all SF-36 domains with GDS. Physical activity programs that promote the improvement or maintenance of physical abilities can positively contribute to the depressive symptomatology and quality of life of the elderly, both through social engagement and physical stimulation. 


\section{keywords}

Elderly. Physical Aptitude. Quality of Life. Depression.

referências

ALMEIDA, Osvaldo; ALMEIDA, Shirley. Confiabilidade da versão brasileira da Escala de Depressão Geriátrica (GDS) versão reduzida. Arquivos de Neuro-Psiquiatria, São Paulo, v. 57, n. 2B, p. 421-426, 1999.

CICONELLI, Rozana. Tradução para o português e validação do questionário genérico de avaliação de qualidade de vida "Medical Outcomes Study 36-Item Short-Form Health Survey (SF-36)". 1997. 148 p. Tese (Doutorado em Medicina) - Escola Paulista de Medicina, Universidade Federal de São Paulo, São Paulo, 1997.

FARIAS, Rosemeri. Qualidade de vida e envelhecimento ativo em idosos mais idosos residentes na comunidade. 2010. Dissertação (Mestrado em Enfermagem) - Universidade Federal de Santa Catarina, Florianópolis, 2010.

FERREIRA, Olivia et al. O envelhecimento ativo sob o olhar de idosos funcionalmente independentes. Revista da Escola de Enfermagem da USP, São Paulo, v. 44, n. 4 , p. 1065-1069, 2010.

FERRETTI, Fátima et al. Análise da qualidade de vida em idosos praticantes e não praticantes de exercício físico regular. Estudos Interdisciplinares sobre o Envelhecimento, Porto Alegre, v. 20, n. 3, p. 729-743, 2015.

GONÇALVES, Andréa et al. Qualidade de vida e sintomas depressivos em idosos de três faixas etárias praticantes de atividade física. Kairós Gerontologia, São Paulo, v. 17, n. 3, p. 79-94, 2014.

GUIMARÃES, Adriana et al. Percepção da qualidade de vida e da finitude de adultos de meia idade e idoso praticantes e não praticantes de atividade física. Revista Brasileira de Geriatria e Gerontologia, Rio de Janeiro, v. 15, n. 4, p. 661-670, 2012.

HAIDER, Sandra et al. Associations between daily physical activity, handgrip strength, muscle mass, physical performance and quality of life in prefrail and frail community-dwelling older adults. Quality of Life Research, New York, v. 25, n. 12, p. 3129-3138, 2016

INSTITUTO BRASILEIRO DE GEOGRAFIA E ESTATÍSTICA (IBGE). Indicadores sociodemográficos e de saúde no Brasil. Rio de Janeiro: IBGE, 2010. Disponível em: http://www.ibge.gov.br. Acesso em: 18 ago. 2018.

LAGO, Laura; REIS, Luciana. Relação entre a qualidade de vida e desempenho motor em idosos ativos. Fisioterapia Brasil, São Paulo, v. 18, n. 6, p. 700-708, 2017.

MACIEL, Erika et al. The relationship between physical aspects of quality of life and extreme levels of regular physical activity in adults. Cadernos de Saúde Pública, Rio de Janeiro, v. 29, n. 11, p. 2251-2260, 2013.

MATHER, Anne et al. Effects of exercise on depressive symptoms in older adults with poorly responsive depressive disorder Randomised controlled trial. The British Journal of Psychiatry, Cambridge, v. 180, n. 5, p. 411-415, 2002.

MAZO, Giovana et al. Autoestima e depressão em idosos praticantes de exercícios físicos. Kinesis, Santa Maria, v. 30, n. 1, 2012.

MERQUIADES, Jucicleide et al. A importância do exercício físico para a qualidade de vida dos idosos. Revista Brasileira de Prescrição e Fisiologia do Exercício, São Paulo, v. 3, n. 18 , p. 597-614, 2009 . 
MORAES, Helena et al. O exercício físico no tratamento da depressão em idosos: revisão sistemática. Revista de Psiquiatria do Rio Grande do Sul, Porto Alegre, v. 29, n. 1, p. 70-79, 2007.

NASCIMENTO, Amanda et al. Experiência subjetiva de idosas durante exercício em ambiente virtual. Motriz, Rio Claro, v. 19, n. 3, p. 68-75, 2013.

NASCIMENTO, Carla et al. Physical exercises, functional capacity and depressive symptoms in brazilian elderly. Revista Brasileira de Cineantropometria e Desempenho Humano, Florianópolis, v. 15, n. 4, p. 486-497, 2013.

OLIVEIRA, Aldalan et al. Qualidade de vida em idosos que praticam atividade física: uma revisão sistemática. Revista Brasileira de Geriatria e Gerontologia, Rio de Janeiro, v. 13, n. 2, p. 301-312, 2010

PASCHOAL, Sérgio. Qualidade de vida na velhice. In: Freitas, Elizabete et al. Tratado de geriatria e gerontologia. 3. ed. Rio de Janeiro: Guanabara Koogan, 2011. p. 99-106.

PATROCINIO, Wanda; TORRES, Stella; GUARIENTO, Maria. Programa de educação popular em saúde: hábitos de vida e sintomas depressivos em idosos. Revista Brasileira de Geriatria e Gerontologia, Rio de Janeiro, v. 16, n. 4, p. 781-792, 2013.

PEREIRA, Déborah; NOGUEIRA, Júlia; SILVA, Carlos. Quality of life and the health status of elderly persons: a population-based study in the central sertão of Ceará. Revista Brasileira de Geriatria e Gerontologia, Rio de Janeiro, v. 18, n. 4, p. 893-908, 2015.

QUEIROZ, Lícia et al. Quality of life and impact of physical activity time in the health of elderly. Revista da Faculdade de Ciências Médicas de Sorocaba, Sorocaba, v. 18, n. 1, p. 24-29, 2016

RIKLLI, Roberta; JONES, Jessie. Development and validation of a functional fitness test for community residing older adults. Journal of Aging and Physical Activity, Birmingham, v. 7, n. 2, p. 129-161, 1999

SHEIKH, Javaid; YESAVAGE, Jerome. Geriatric Depression Scale (GDS): recent evidence and development of a shorter version. Clinical Gerontologist: the Journal of Aging and Mental Health, London, v. 5, n. 1-2, p. 165-173, 1986.

SILVA, Denise. Papel do grupo de ginástica no processo do envelhecimento. Revista Portal de Divulgação, São Paulo, v. 37, n. 4, 2013.

SILVEIRA, Michele et al. Atividade física e qualidade de vida em idosos. Saúde e Pesquisa, Maringá, v. 4, n. 3, p. 417-424, 2011.

STELLA, Florindo et al. Depressão no idoso: diagnóstico, tratamento e benefícios da atividade física. Motriz, Rio Claro, v. 8, n. 3, p. 91-98, 2002.

TOSCANO, José; OLIVEIRA, Antônio. Qualidade de vida em idosos com distintos níveis de atividade física. Revista Brasileira de Medicina do Esporte, São Paulo, v. 15, n. 3, p. 169-173, 2009.

VILA, Camilla et al. Aptidão física funcional e nível de atenção em idosas praticantes de exercício físico. Revista Brasileira de Geriatria e Gerontologia, Rio de Janeiro, v. 16, n. 2 , p. 355-364, 2013.

WORLD HEALTH ORGANIZATION (WHO). Whoqol-bref: introduction, administration, scoring and generic version of the assessment: field trial version. Geneva: WHO, 1996. 